\title{
Multiple Homoclinic Solutions for a Class of Superquadratic Fourth-order Differential Equations
}

\author{
Mohsen Timoumi \\ Faculty of Sciences.Dpt of Mathematics, 5000 Monastir. Tunisia \\ m_timoumi@yahoo.com
}

\begin{abstract}
Applying a Symmetric Mountain Pass Theorem, we prove the existence of infinitely many homoclinic solutions for a class of fourth-order differential equations

$$
u^{(4)}(x)+\omega u^{\prime \prime}(x)+a(x) u(x)=f(x, u(x)), \forall x \in \mathbb{R}
$$

where $a \in C(\mathbb{R}, \mathbb{R})$ may be negative on a bounded interval and $F(x, u)=\int_{0}^{u} f(x, t) d t$ is superquadratic at infinity in the second variable but does not need to satisfy the well-known Ambrosetti-Rabinowitz superquadratic growth condition.
\end{abstract}

Keywords: Fourth-order differential equation; homoclinic solutions; variational methods; Symmetric Mountain Pass Theorem.

MSC2010 58E05; 34C37; 70H05.

\section{Introduction}

We consider the nonperiodic fourth-order differential equation

$$
u^{(4)}(x)+\omega u^{\prime \prime}(x)+a(x) u(x)=f(x, u(x)), \forall x \in \mathbb{R}
$$

where $\omega$ is a constant, $a \in C(\mathbb{R}, \mathbb{R})$ and $f \in C(\mathbb{R} \times \mathbb{R}, \mathbb{R})$. It is well-known that the mathematical modeling of important questions in different fields of research such as mechanical engineering, control systems, economics and many others, leads naturally to the consideration of the nonlinear differential equations. In particular, the fourth order differential equations, like (1.1) have been put forward as mathematical model for the study of pattern formation in physics and mechanics. For example the well-known extended Fisher-Kolmogorov equation proposed by Coullet et al. in 1987 [5], in the study of phase transitions, the fourth-order elastic beam equation in describing a large class of elastic deflection [14], the Swift-Hohenberg equation which is a general model for pattern-forming process derived in [6] to describe random thermal fluctuations in the Boussinesque equation and in the propagation of lasers [7].

As usual, we say that a solution $u$ of equation (1.1) is homoclinic (to 0) if $u(x) \rightarrow 0$ as $x \longrightarrow \pm \infty$. In addition, if $u \neq 0$, then $u$ is called a nontrivial homoclinic solution.

During the previous years, the existence and multiplicity of homoclinic solutions for (1.1) have been extensively investigated via critical point theory and variational methods. From the beginning, most of them treated the case where $a(x)$ and $f(x, u)$ are either independent of $x$ or periodic in $x$, see $[1,3,4,8,16]$ and the references cited therein. In this kind of problems, the function $a$ plays an important role. Compared with the case of $a(x)$ and $f(x, u)$ being periodic in $x$, there is less literature available for the case where $a(x)$ and $f(x, u)$ are nonperiodic in $x$, see [9-13,17-20]. We notice that, for the case that equation (1.1) is not periodic, to obtain the existence of homoclinic solutions, the following coercive condition on $a$ is often needed:

$\left(\mathcal{A}_{0}\right) a: \mathbb{R} \longrightarrow \mathbb{R}$ is a continuous function, and there exists a constant $r_{0}$ such that

$$
0<r_{0} \leq a(x) \longrightarrow+\infty \text { as }|x| \longrightarrow \infty
$$


and

$$
\omega \leq 2 \sqrt{r_{0}}
$$

which is used to establish the corresponding compact embedding lemmas on suitable functional spaces, see $[9,10,17,18]$. Most of these known results were obtained for the case where $F$ is superquadratic at infinity in $u$ and satisfies the usual assumption:

$$
\lim _{|u| \longrightarrow 0} \frac{F(x, u)}{|u|^{2}}=0 \text { uniformly for } t \in \mathbb{R} .
$$

In this case, the well-known Ambrosetti-Rabinowitz superquadratic condition was usually assumed on $F$, see $[8,13,16,20]$. In this paper, we study the existence of infinitely many homoclinic solutions for (1.1) in the case where $a$ is unnecessarily required to be positive, and $F$ satisfies some weak superquadratic conditions at infinity with respect to $u$. More precisely, we make the following assumptions:

$$
\lim _{|x| \longrightarrow \infty} a(x)=+\infty
$$

$\left(F_{1}\right)$ There exist constants $a_{0}, R_{0}>0$ such that

$$
|f(x, u)| \leq a_{0}|u|, \forall(x, u) \in \mathbb{R} \times \mathbb{R} \text { with }|u| \leq R_{0} ;
$$

$$
\lim _{|u| \rightarrow+\infty} \frac{|F(x, u)|}{|u|^{2}}=+\infty, \forall x \in \mathbb{R}
$$

and

$$
F(x, u) \geq 0, \forall(x, u) \in \mathbb{R} \times \mathbb{R} \text { with }|u| \geq R_{0}
$$

$$
F(x,-u)=F(x, u), \forall(x, u) \in \mathbb{R} \times \mathbb{R}
$$

$\left(F_{4}\right)$ There exist $g \in L^{1}(\mathbb{R})$ and constants $b_{0}, c_{0}>0$ and $\left.\nu \in\right] 0,2[$ such that

$$
\begin{gathered}
\widehat{F}(x, u)=\frac{1}{2} f(x, u) u-F(x, u) \geq\left\{\begin{array}{l}
g(x), \forall x \in \mathbb{R},|x| \leq R_{0} \\
b_{0}|u|^{\nu}, \forall x \in \mathbb{R},|u| \geq R_{0} ;
\end{array}\right. \\
|F(x, u)| \leq c_{0}|u|^{2-\nu} \widehat{F}(x, u), \forall(x, u) \in \mathbb{R} \times \mathbb{R} \text { with }|u| \geq R_{0} ;
\end{gathered}
$$

$\left(F_{5}\right)$ There exist constants $\mu>2$ and $\rho>0$ such that

$$
\mu F(x, u) \leq f(x, u) u+\rho|u|^{2}, \forall(x, u) \in \mathbb{R} \times \mathbb{R} .
$$

Our main results read as follows.

Theorem 1.1. Assume that $(\mathcal{A})$ and $\left(F_{1}\right)-\left(F_{4}\right)$ are satisfied. Then $(\mathcal{F O D} \mathcal{E})$ possesses infinitely many nontrivial homoclinic orbits.

Theorem 1.2. Assume that $(\mathcal{A}),\left(F_{1}\right)-\left(F_{3}\right)$ and $\left(F_{5}\right)$ are satisfied. Then $(\mathcal{F} \mathcal{O D} \mathcal{E})$ possesses infinitely many nontrivial homoclinic orbits.

Remarks 1.1. a) In our results, $a(x)$ is unnecessarily positive. For example $a(x)=|x|-1$ satisfies $(\mathcal{A})$ but it does'nt satisfy $\left(\mathcal{A}_{0}\right)$.

b) Let $F(x, u)=\chi(x)|x|^{2} \ln \left(\frac{1}{2}+|x|\right)$, where $\chi$ is a continuous bounded function with positive lower bound. Then an easy computation shows that $F$ satisfies the superquadratic conditions $\left(F_{1}\right)-\left(F_{4}\right)$. However, $F$ does not satisfy the $(\mathcal{A R})$-condition.

The remainder of this paper is organized as follows. In Section 2, some preliminary results are presented. Section 3 is devoted to the proofs of our results. 


\section{Variational setting and preliminaries}

To prove our main result via critical point theory, we need to establish the variational setting for (1.1). In the following, we shall use $\|\cdot\|_{s}$ to denote the norm of $L^{s}(\mathbb{R})$ for any $s \in[2, \infty]$. Let $H^{2}(\mathbb{R})$ be the Sobolev space with inner product and norm given respectively by

$$
<u, v>_{H^{2}}=\int_{\mathbb{R}}\left[u^{\prime \prime}(x) v^{\prime \prime}(x)+u^{\prime}(x) v^{\prime}(x)+u(x) v(x)\right] d x
$$

and

$$
\|u\|_{H^{2}}=<u, u>_{H^{2}}^{\frac{1}{2}}
$$

for all $u, v \in H^{2}(\mathbb{R})$.

Lemma 2.1 ([4, Lemma 8]). Assume that $a$ satisfies $\left(\mathcal{A}_{0}\right)$. Then there exists a constant $b>0$ such that

$$
\int_{\mathbb{R}}\left[u^{\prime \prime}(x)^{2}-\omega u^{\prime}(x)^{2}+a(x) u(x)^{2}\right] d x \geq b\|u\|_{H^{2}}^{2}, \forall u \in H^{2}(\mathbb{R}) .
$$

By Lemma 2.1, we define

$$
E=\left\{u \in H^{2}(\mathbb{R}) / \int_{\mathbb{R}}\left[u^{\prime \prime}(x)^{2}-\omega u^{\prime}(x)^{2}+a(x) u(x)^{2}\right] d x<\infty\right\}
$$

with the inner product

$$
<u, v>=\int_{\mathbb{R}}\left[u^{\prime \prime}(x) v^{\prime \prime}(x)-\omega u^{\prime}(x) v^{\prime}(x)+a(x) u(x) v(x)\right] d x
$$

and the corresponding norm

$$
\|u\|=\left(\int_{\mathbb{R}}\left[u^{\prime \prime}(x)^{2}-\omega u^{\prime}(x)^{2}+a(x) u(x)^{2}\right] d x\right)^{\frac{1}{2}} .
$$

It is easy to verify that $E$ is a Hilbert space.

In order to prove our result, the following compactness result is necessary.

Lemma 2.2 ([17, Lemma 2.2]). Assume that $a$ satisfies $\left(\mathcal{A}_{0}\right)$. Then $E$ is compactly embedded in $L^{s}(\mathbb{R})$ for all $s \in[2, \infty]$. Moreover, for all $s \in[2, \infty]$, there exists $\eta_{s}>0$ such that

$$
\|u\|_{L^{s}(\mathbb{R})} \leq \eta_{s}\|u\|, \forall u \in E .
$$

To study the critical points of the variational functional associated with $(\mathcal{F O D} \mathcal{E})$, we need to recall the Symmetric Mountain Pass Theorem [15].

Definition 2.1. Let $E$ be a Banach space with the norm $\|$.$\| , we say that \Phi \in C^{1}(E, \mathbb{R})$ satisfies the

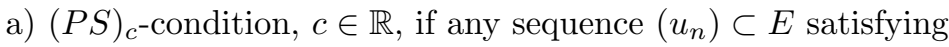

$$
\Phi\left(u_{n}\right) \longrightarrow \text { c and } \Phi^{\prime}\left(u_{n}\right) \longrightarrow 0 \text { as } n \longrightarrow \infty
$$

possesses a convergent subsequence,

b) $(C)_{c}-$ condition, $c \in \mathbb{R}$, if any sequence $\left(u_{n}\right) \subset E$ satisfying

$$
\Phi\left(u_{n}\right) \longrightarrow c \text { and }\left\|\Phi^{\prime}\left(u_{n}\right)\right\|\left(1+\left\|u_{n}\right\|\right) \longrightarrow 0 \text { as } n \longrightarrow \infty
$$

possesses a convergent subsequence.

Lemma 2.3. Let $E$ be an infinite dimensional Banach space, $E=Y \oplus Z$, where $Y$ is finite dimensional space. Suppose that $\Phi \in C^{1}(E, \mathbb{R})$ satisfies the $(P S)_{c}-$ condition and and

$$
\Phi(0)=0, \Phi(-u)=\Phi(u), \forall u \in E
$$


(b) There exist constants $\rho, \alpha>0$ such that $\Phi_{\mid \partial B_{\rho} \cap Z} \geq \alpha$;

(c) For any finite dimensional subspace $\widetilde{E} \subset E$, there is $R=R(\widetilde{E})>0$ such that $\Phi(u) \leq 0$ on $\widetilde{E} \backslash B_{R}$, where $B_{R}=\{u \in E /\|u\|<R\}$.

Then $\Phi$ possesses an unbounded sequence of critical values.

Remark 2.1. As shown in [2], a deformation lemma can be proved with $(C)_{c}$-condition replacing the $(P S)_{c}-$ condition, and it turns out that Lemma 2.3 still holds true with the $(C)_{c}$-condition instead of the $(P S)_{c}-$ condition.

\section{Proof of Theorems}

From $(\mathcal{A}),\left(F_{1}\right)$ and $\left(F_{2}\right)$, we know that there exists a positive constant $r_{0}$ such that $a(x)+2 r_{0} \geq r_{0}$ for all $x \in \mathbb{R}$. Let $\bar{a}(x)=a(x)+2 r_{0}$ and $\bar{F}(x, u)=F(x, u)+r_{0}|u|^{2}$. Consider the following fourth-order differential equation

$$
u^{(4)}(x)+\omega u^{\prime \prime}(x)+\bar{a}(x) u(x)=\bar{f}(x, u(x)), \forall x \in \mathbb{R},
$$

then $(\overline{\mathcal{F O D} \mathcal{E}})$ is equivalent to $(\mathcal{F O D} \mathcal{E})$. Moreover, it is easy to check that the hypotheses $\left(F_{1}\right)-\left(F_{5}\right)$ still hold for $\bar{F}$ provided that those hold for $F$, and $\bar{a}$ satisfies the condition $\left(\mathcal{A}_{0}\right)$. Hence, in what follows, we always assume without loss of generality that $a$ satisfies $\left(\mathcal{A}_{0}\right)$ instead of $(\mathcal{A})$.

Consider the variational functional $\Phi$ associated to $(\mathcal{F O} \mathcal{O} \mathcal{E})$ :

$$
\Phi(u)=\frac{1}{2} \int_{\mathbb{R}}\left[u^{\prime \prime}(x)^{2}-\omega u^{\prime}(x)^{2}+a(x) u(x)^{2}\right] d x-\int_{\mathbb{R}} F(x, u(x)) d x
$$

defined on the space $E$ introduced in Section 2.

Lemma 3.1. Under assumptions $\left(\mathcal{A}_{0}\right)$ and $\left(F_{1}\right)$, the functional

$$
\psi(u)=\int_{\mathbb{R}} F(x, u) d x
$$

is continuously differentiable on $E$ and

$$
\psi^{\prime}(u) v=\int_{\mathbb{R}} f(x, u) \cdot v d x, \forall u, v \in E .
$$

Proof. For any given $u \in E$, we know that $u \in H^{2}(\mathbb{R})$ and hence there exists a constant $R>0$ such that

$$
|u(x)| \leq \frac{R_{0}}{2}, \forall|x| \geq R
$$

By (2.1), for any $v \in E$ with $\|v\| \leq \frac{R_{0}}{2 \eta_{\infty}}$, we have

$$
\|v\|_{L^{\infty}} \leq \frac{R_{0}}{2}
$$

Combining (3.2), (3.3) and $\left(F_{1}\right)$, by the Mean Value Theorem and Hölder's inequality, for any $r \geq R$ and $v \in E$ with $\|v\| \leq \frac{R_{0}}{2 \eta_{\infty}}$, one has

$$
\begin{array}{r}
\left|\int_{|x| \geq r}[F(x, u+v)-F(x, u)-f(x, u) v] d x\right| \\
=\left|\int_{|x| \geq r} \int_{0}^{1}(f(x, u+s v)-f(x, u)) v d s d x\right| \\
\leq 2 a_{0} \int_{|x| \geq r}(|u|+|v|)|v| d x \leq 2 a_{0}\left(\int_{|x| \geq r}(|u|+|v|)^{2} d x\right)^{\frac{1}{2}}\|v\|_{L^{2}}
\end{array}
$$




$$
\leq 2 a_{0} \eta_{2}\left[\left(\int_{|x| \geq r}|u|^{2} d x\right)^{\frac{1}{2}}+\eta_{2}\|v\|\right]\|v\|
$$

Since $u \in L^{2}(\mathbb{R})$, for any $\epsilon>0$, there exist $0<\alpha_{1}<\frac{R_{0}}{2 \eta_{\infty}}$ and $R_{\epsilon} \geq R$ such that for all $v \in E$ with $\|v\| \leq \alpha_{1}$

$$
2 a_{0} \eta_{2}\left[\left(\int_{|x| \geq r}|u|^{2} d x\right)^{\frac{1}{2}}+\eta_{2}\|v\|\right] \leq \frac{\epsilon}{2} .
$$

It is well known that the functional

$$
\psi_{\epsilon}(u)=\int_{\left[-R_{\epsilon}, R_{\epsilon}\right]} F(x, u) d x
$$

is continuously differentiable on $H^{2}\left(\left[-R_{\epsilon}, R_{\epsilon}\right]\right)$. Thus, since $E$ is compactly embedded in $H^{2}(\mathbb{R})$, there exists a constant $\alpha_{2}>0$ such that for all $\|v\| \leq \alpha_{2}$

$$
\left|\int_{\left[-R_{\epsilon}, R_{\epsilon}\right]}[F(x, u+v)-F(x, u)-f(x, u) v] d x\right| \leq \frac{\epsilon}{2}\|v\| .
$$

Taking $\alpha=\min \left\{\alpha_{1}, \alpha_{2}\right\}$, then (3.4)-(3.6) imply

$$
\left|\int_{\mathbb{R}}[F(x, u+v)-F(x, u)-f(x, u) v] d x\right| \leq \epsilon\|v\|
$$

for all $v \in E$ with $\|v\| \leq \alpha$. Therefore, $\psi$ is differentiable on $E$ and satisfies (3.1).

It remains to prove that $\psi^{\prime}$ is continuous. Let $u_{n} \longrightarrow u$ in $E$. By Hölder's inequality, we have

$$
\begin{gathered}
\left\|\psi^{\prime}\left(u_{n}\right)-\psi^{\prime}(u)\right\|_{E^{\prime}}=\sup _{\|v\|=1}\left|\psi^{\prime}\left(u_{n}\right) v-\psi^{\prime}(u) v\right| \\
=\sup _{\|v\|=1}\left|\int_{\mathbb{R}}\left[f\left(x, u_{n}\right)-f(x, u)\right] v d x\right| \\
\leq \sup _{\|v\|=1}\left(\int_{\mathbb{R}}\left|f\left(x, u_{n}\right)-f(x, u)\right|^{2} d x\right)^{\frac{1}{2}}\|v\|_{L^{2}} \\
\leq \eta_{2}\left(\int_{\mathbb{R}}\left|f\left(x, u_{n}\right)-f(x, u)\right|^{2} d x\right)^{\frac{1}{2}} .
\end{gathered}
$$

Lemma 2.1 implies that $u_{n} \longrightarrow u$ in $L^{s}(\mathbb{R})$ for $s=1$ and $s=\infty$. Let $M$ be a positive constant such that $\left\|u_{n}\right\|_{L^{1}} \leq M$ for all integer $n$. By $\left(F_{1}\right)$, for any $\epsilon>0$, there exists a constant $0<r<R_{0}$ such that for all $x \in \mathbb{R}$ and $|u| \leq r$

$$
|f(x, u)| \leq \frac{\epsilon}{2\left(M^{\frac{1}{2}}+\|u\|_{L^{1}}^{\frac{1}{2}}\right)}|u|^{\frac{1}{2}} .
$$

Due to $(3.8)$ and the facts that $u \in H^{2}(\mathbb{R})$ and $u_{n} \longrightarrow u$ in $L^{\infty}(\mathbb{R})$, there exists $R_{\epsilon}>R_{0}$ and $N_{1} \in \mathbb{N}$ such that for all $|x| \geq R_{\epsilon}$ and $n \geq N_{1}$

$$
\begin{aligned}
\left|f\left(x, u_{n}(x)\right)\right| & \leq \frac{\epsilon}{2\left(M^{\frac{1}{2}}+\|u\|_{L^{1}}^{\frac{1}{2}}\right)}\left|u_{n}(x)\right|^{\frac{1}{2}} \\
|f(x, u(x))| & \leq \frac{\epsilon}{2\left(M^{\frac{1}{2}}+\|u\|_{L^{1}}^{\frac{1}{2}}\right)}|u(x)|^{\frac{1}{2}} .
\end{aligned}
$$

Thus

$$
\begin{gathered}
\left(\int_{|t| \geq R_{\epsilon}}\left|f\left(x, u_{n}\right)-f(x, u)\right|^{2} d x\right)^{\frac{1}{2}} \\
\leq\left(\int_{|x| \geq R_{\epsilon}}\left|f\left(x, u_{n}\right)\right|^{2} d x\right)^{\frac{1}{2}}+\left(\int_{|x| \geq R_{\epsilon}}|f(x, u)|^{2} d x\right)^{\frac{1}{2}}
\end{gathered}
$$




$$
\leq \frac{\epsilon}{2\left(M^{\frac{1}{2}}+\|u\|_{L^{1}}^{\frac{1}{2}}\right)}\left(\left\|u_{n}\right\|_{L^{1}}^{\frac{1}{2}}+\|u\|_{L^{1}}^{\frac{1}{2}}\right) \leq \frac{\epsilon}{2} .
$$

Observing that $u_{n} \longrightarrow u$ in $L^{\infty}(\mathbb{R})$, then by Lebesgue's Dominated Convergence Theorem, we have

$$
\left(\int_{\left[-R_{\epsilon}, R_{\epsilon}\right]}\left|f\left(x, u_{n}\right)-f(x, u)\right|^{2} d x\right)^{\frac{1}{2}} \longrightarrow 0 \text { as } n \longrightarrow \infty .
$$

Hence, there is $N_{2} \in \mathbb{N}$ such that for all $n \geq N_{2}$

$$
\left(\int_{\left[-R_{\epsilon}, R_{\epsilon}\right]}\left|f\left(x, u_{n}\right)-f(x, u)\right|^{2} d x\right)^{\frac{1}{2}} \leq \frac{\epsilon}{2},
$$

which together with (3.9) implies that for all $n \geq \max \left\{N_{1}, N_{2}\right\}$

$$
\left(\int_{\mathbb{R}}\left|f\left(x, u_{n}\right)-f(x, u)\right|^{2} d x\right)^{\frac{1}{2}} \leq \epsilon .
$$

Combining this with (3.7) implies that $\psi^{\prime}\left(u_{n}\right) \longrightarrow \psi^{\prime}(u)$ as $n \longrightarrow \infty$ and then $\psi \in C^{1}(E, \mathbb{R})$. The proof of Lemma 3.1 is completed.

From Lemma 3.1, we deduce that $\Phi \in C^{1}(E, \mathbb{R})$ and

$$
\Phi^{\prime}(u) v=<u, v>-\int_{\mathbb{R}} f(x, u) \cdot v d x, \forall u, v \in E .
$$

Moreover, we can infer that $u \in E$ is a critical point of $\Phi$ if and only if it is a (classical) solution of problem $(\mathcal{F O D E})$.

Lemma 3.2. Under assumptions $\left(\mathcal{A}_{0}\right),\left(F_{1}\right)$ and $\left(F_{2}\right)$, for any finite dimensional subspace $\widetilde{E} \subset E$, there is $R=R(\widetilde{E})>0$ such that

$$
\Phi(u) \leq 0, \forall u \in \widetilde{E},\|u\| \geq R
$$

Proof. We will prove the following

$$
\Phi(u) \longrightarrow-\infty \text { as }\|u\| \longrightarrow \infty, u \in \widetilde{E} .
$$

Arguing indirectly, assume that there exists a sequence $\left(u_{n}\right) \subset \widetilde{E}$ with $\left\|u_{n}\right\| \longrightarrow \infty$ and a constant $M>0$ such that $\Phi\left(u_{n}\right) \geq-M$ for all $n \in \mathbb{N}$. Set $v_{n}=\frac{u_{n}}{\left\|u_{n}\right\|}$, then $\left\|v_{n}\right\|=1$. Passing to a subsequence if necessary, we may assume that $v_{n} \rightarrow v$ in $E$. Since $\widetilde{E}$ is finite dimensional, we have $v_{n} \longrightarrow v$ in $\widetilde{E}$ and $v_{n} \longrightarrow v$ a.e. on $\mathbb{R}$. It follows that $\|v\|=1$. For $0 \leq a<b$, let

$$
\begin{aligned}
\Omega_{n}(a, b) & =\left\{x \in \mathbb{R} / a \leq\left|u_{n}(x)\right|<b\right\} \\
A & =\{x \in \mathbb{R} / v(x) \neq 0\} .
\end{aligned}
$$

Since $v \neq 0$, then meas $(A)>0$. For a.e. $t \in \mathbb{R}$, we have $\lim _{n \rightarrow \infty}\left|u_{n}(x)\right|=\infty$, hence $x \in \Omega_{n}\left(R_{0}, \infty\right)$ for $n$ large enough. Since $v_{n}(x) \longrightarrow v(x)$ a.e. $x \in \mathbb{R}$, we have $\chi_{\Omega_{n}\left(R_{0}, \infty\right)}(x)\left|v_{n}(x)\right| \longrightarrow|v(x)|$ a.e. $x \in A$, where $\chi_{\Omega}$ denotes the characteristic function of $\Omega$. Hence, it follows from $\left(F_{1}\right),\left(F_{2}\right)$ and Fatou's Lemma that

$$
\begin{gathered}
0=\lim _{n \longrightarrow \infty} \frac{-M}{\left\|u_{n}\right\|^{2}} \leq \lim _{n \longrightarrow \infty} \frac{\Phi\left(u_{n}\right)}{\left\|u_{n}\right\|^{2}}=\lim _{n \longrightarrow \infty}\left[\frac{1}{2}-\int_{\mathbb{R}} \frac{F\left(x, u_{n}\right)\left|v_{n}\right|^{2}}{\left|u_{n}\right|^{2}} d x\right] \\
=\lim _{n \rightarrow \infty}\left[\frac{1}{2}-\int_{\Omega_{n}\left(0, R_{0}\right)} \frac{F\left(x, u_{n}\right)\left|v_{n}\right|^{2}}{\left|u_{n}\right|^{2}} d x-\int_{\Omega_{n}\left(R_{0}, \infty\right)} \frac{F\left(x, u_{n}\right)\left|v_{n}\right|^{2}}{\left|u_{n}\right|^{2}} d x\right] \\
\quad \leq \limsup _{n \longrightarrow \infty}\left[\frac{1}{2}+\frac{a_{0}}{2} \int_{\mathbb{R}}\left|v_{n}\right|^{2} d x-\int_{\Omega_{n}\left(R_{0}, \infty\right)} \frac{F\left(x, u_{n}\right)\left|v_{n}\right|^{2}}{\left|u_{n}\right|^{2}} d x\right]
\end{gathered}
$$




$$
\begin{gathered}
\leq \frac{1}{2}+\frac{a_{0}}{2} \eta_{2}^{2}-\liminf _{n \rightarrow \infty} \int_{\Omega_{n}\left(R_{0}, \infty\right)} \frac{F\left(x, u_{n}\right)\left|v_{n}\right|^{2}}{\left|u_{n}\right|^{2}} d x \\
\leq \frac{1}{2}+\frac{a_{0}}{2} \eta_{2}^{2}-\int_{\mathbb{R}} \liminf _{n \rightarrow \infty} \frac{F\left(x, u_{n}\right)\left|v_{n}\right|^{2}}{\left|u_{n}\right|^{2}} \chi_{\mid \Omega_{n}\left(R_{0}, \infty\right)}(x) d x=-\infty
\end{gathered}
$$

which is a contradiction. Hence (3.13) and then (3.12) is verified. The proof of Lemma 3.2 is completed.

Let $\left(e_{j}\right)_{j \in \mathbb{N}}$ be an orthonormal basis of $E$ and define $X_{j}=\mathbb{R} e_{j}$

$$
Y_{k}=\oplus_{j=1}^{k} X_{j}, Z_{k}=\overline{\oplus_{j=k+1}^{\infty} X_{j}}, k \in \mathbb{N} .
$$

Lemma 3.3. Suppose $\left(\mathcal{A}_{0}\right)$ hold. Then for any $p \in[2, \infty]$

$$
l_{p}(k)=\sup _{u \in Z_{k},\|u\|=1}\|u\|_{L^{p}} \longrightarrow 0 \text { as } k \longrightarrow \infty .
$$

Proof. It is clear that $0<l_{p}(k+1) \leq l_{p}(k)$, so that $l_{p}(k) \longrightarrow \bar{l}_{p}$ as $k \longrightarrow \infty$. For every $k \geq 1$, there exists $u_{k} \in Z_{k}$ such that $\left\|u_{k}\right\|=1$ and $\left\|u_{k}\right\|_{L^{p}}>\frac{1}{2} l_{p}(k)$. For any $v \in E$, let $v=\sum_{i=1}^{\infty} v_{i} e_{i}$. By the Cauchy-Schwartz inequality, one has

$$
\begin{aligned}
& \left|<u_{k}, v>\right|=\left|<u_{k}, \sum_{i=1}^{\infty} v_{i} e_{i}\right\rangle|=|<u_{k}, \sum_{i=k+1}^{\infty} v_{i} e_{i}>\mid \\
& \leq\left\|u_{k}\right\|\left\|\sum_{i=k+1}^{\infty} v_{i} e_{i}\right\| \leq \sum_{i=k+1}^{\infty}\left|v_{i}\right|\left\|e_{i}\right\| \longrightarrow 0 \text { as } k \longrightarrow \infty
\end{aligned}
$$

which implies that $u_{k} \rightarrow 0$. Without loss of generality, Lemma 2.2 implies that $u_{k} \longrightarrow 0$ in $L^{2}(\mathbb{R})$. Thus we have proved that $\bar{l}_{p}=0$. The proof of Lemma 3.3 is completed.

By (3.15), we can choose an integer $m \geq 1$ such that

$$
\|u\|_{L^{p}} \leq \frac{1}{2 a_{0}}\|u\|, \forall u \in Z_{m} .
$$

In the following, we will apply Lemma 2.2 with $Y=Y_{m}$ and $Z=Z_{m}$.

Lemma 3.4. Under assumptions $\left(\mathcal{A}_{0}\right)$ and $\left(F_{1}\right)$, there exist constants $\rho, \alpha>0$ such that $\Phi_{\mid \partial B_{\rho} \cap Z} \geq \alpha$.

Proof. If $\|u\|=\frac{R_{0}}{\eta_{\infty}}$, then by Lemma 2.2, we have $\|u\|_{L^{\infty}} \leq R_{0}$. Hence, it follows from $\left(F_{1}\right)$ that

$$
F(x, u(x)) \leq \frac{a_{0}}{2}|u(x)|^{2}, \forall u \in E,\|u\|=\frac{R_{0}}{\eta_{\infty}} .
$$

Combining (3.16) with (3.17) yields for all $u \in Z$ with $\|u\|=\frac{R_{0}}{\eta_{\infty}}=\rho$,

$$
\begin{gathered}
\Phi(u)=\frac{1}{2}\|u\|^{2}-\int_{\mathbb{R}} F(x, u) d x \\
\geq \frac{1}{2}\|u\|^{2}-\frac{a_{0}}{2} \int_{\mathbb{R}}|u|^{2} d x \geq \frac{1}{2}\|u\|^{2}-\frac{a_{0}}{2}\|u\|_{L^{2}}^{2} \\
\geq \frac{1}{4}\|u\|^{2}=\frac{1}{4}\left(\frac{R_{0}}{\eta_{\infty}}\right)^{2}=\alpha .
\end{gathered}
$$

The proof of Lemma 3.4 is completed.

Proof of Theorem 1.1. By assumptions $\left(F_{1}\right)$ and $\left(F_{3}\right)$, it is clear that

$$
\Phi(0)=0 \text { and } \Phi(-u)=\Phi(u), \forall u \in E .
$$


Thus the condition (a) of Lemma 2.3 is satisfied. Lemmas 3.2, 3.4 imply that conditions (b) and (c) of Lemma 2.3 are satisfied. It remains to prove that $\Phi$ satisfies the $(C)_{c}-$ condition.

Lemma 3.5. Assume that $\left(\mathcal{A}_{0}\right),\left(F_{1}\right),\left(F_{2}\right)$ and $\left(F_{4}\right)$ are satisfied. Then $\Phi$ verifies the $(C)_{c}-$ condition for all $c \in \mathbb{R}$.

Proof. Let $\left(u_{n}\right)$ be a $(C)_{c}$ sequence, that is

$$
\Phi\left(u_{n}\right) \longrightarrow \text { and }\left\|\Phi^{\prime}\left(u_{n}\right)\right\|\left(1+\left\|u_{n}\right\|\right) \longrightarrow 0 \text { as } n \longrightarrow \infty .
$$

Firstly, we prove that $\left(u_{n}\right)$ is bounded in $E$. Arguing by contradiction, suppose that $\left\|u_{n}\right\| \longrightarrow \infty$ as $n \longrightarrow \infty$. Let $v_{n}=\frac{u_{n}}{\left\|u_{n}\right\|}$. Then $\left\|v_{n}\right\|=1$. Observe that for $n$ large enough

$$
c+1 \geq \Phi\left(u_{n}\right)-\frac{1}{2} \Phi^{\prime}\left(u_{n}\right) u_{n}=\int_{\mathbb{R}} \widehat{F}\left(x, u_{n}\right) d x
$$

It follows from (3.19) that

$$
\frac{1}{2} \leq \lim \sup _{n \rightarrow \infty} \int_{\mathbb{R}} \frac{\left|F\left(x, u_{n}\right)\right|}{\left\|u_{n}\right\|^{2}} d x .
$$

Passing to a subsequence if necessary, we may assume that $v_{n} \rightarrow v$ in $E$. Then by Lemma 2.2, without loss of generality, we have $v_{n} \longrightarrow v$ in $L^{2}(\mathbb{R})$ and $v_{n} \longrightarrow v$ a.e. on $\mathbb{R}$.

If $v=0$, then $v_{n} \longrightarrow 0$ in $L^{2}(\mathbb{R})$ and $v_{n} \longrightarrow 0$ a.e. on $\mathbb{R}$. Hence, it follows from $\left(F_{1}\right)$ that

$$
\begin{aligned}
& \int_{\Omega_{n}\left(0, R_{0}\right)} \frac{\left|F\left(x, u_{n}\right)\right|}{\left\|u_{n}\right\|^{2}} d x=\int_{\Omega_{n}\left(0, R_{0}\right)} \frac{\left|F\left(x, u_{n}\right)\right|}{\left|u_{n}\right|^{2}}\left|v_{n}\right|^{2} d x \\
& \leq \frac{a_{0}}{2} \int_{\Omega_{n}\left(0, R_{0}\right)}\left|v_{n}\right|^{2} d x \leq \frac{a_{0}}{2}\left\|v_{n}\right\|_{L^{2}}^{2} \longrightarrow 0 \text { as } n \longrightarrow \infty .
\end{aligned}
$$

From $\left(F_{4}\right)$ and $(3.19)$, one has

$$
\begin{gathered}
\int_{\Omega_{n}\left(R_{0}, \infty\right)} \frac{\left|F\left(x, u_{n}\right)\right|}{\left\|u_{n}\right\|^{2}} d x=\int_{\Omega_{n}\left(R_{0}, \infty\right)} \frac{\left|F\left(x, u_{n}\right)\right|}{\left|u_{n}\right|^{2}}\left|v_{n}\right|^{2} d x \\
\leq \frac{c_{0}\left\|v_{n}\right\|_{\infty}^{2-\nu}}{\left\|u_{n}\right\|^{\nu}} \int_{\Omega_{n}\left(R_{0}, \infty\right)} \widehat{F}\left(x, u_{n}\right) d x \\
\leq \frac{c_{0}\left\|v_{n}\right\|_{L^{\infty}}^{2-\nu}}{\left\|u_{n}\right\|^{\nu}}\left[1+c-\int_{\Omega_{n}\left(0, R_{0}\right)} \widehat{F}\left(x, u_{n}\right) d x\right] \\
\leq \frac{c_{0} \eta_{\infty}^{2-\nu}}{\left\|u_{n}\right\|^{\nu}}\left[1+c-\int_{\Omega_{n}\left(0, R_{0}\right)} g(x) d x\right] \\
\leq \frac{c_{0} \eta_{\infty}^{2-\nu}}{\left\|u_{n}\right\|^{\nu}}\left[1+c+\int_{\mathbb{R}}|g(x)| d x\right] \longrightarrow 0 \text { as } n \longrightarrow \infty .
\end{gathered}
$$

Combining (3.22) with (3.23) yields

$$
\int_{\mathbb{R}} \frac{\left|F\left(x, u_{n}\right)\right|}{\left\|u_{n}\right\|^{2}} d x=\int_{\Omega_{n}\left(0, R_{0}\right)} \frac{\left|F\left(x, u_{n}\right)\right|}{\left\|u_{n}\right\|^{2}} d x+\int_{\Omega_{n}\left(R_{0}, \infty\right)} \frac{\left|F\left(x, u_{n}\right)\right|}{\left\|u_{n}\right\|^{2}} d x \longrightarrow 0 \text { as } n \longrightarrow \infty
$$

which contradicts $(3.21)$.

If $v \neq 0$. By a similar fashion as for (3.14), we can get a contradiction. Therefore, $\left(u_{n}\right)$ is bounded in $E$.

Next, we prove that $\left(u_{n}\right)$ possesses a convergent subsequence. Without loss of generality, we can assume by Lemma 
2.2 that $u_{n} \longrightarrow u$ in $L^{s}(\mathbb{R})$ for $s=1$ and $s=\infty$. Using Hölder's inequality, we can show as in the proof of Lemma 3.1 that

$$
\int_{\mathbb{R}}\left[f\left(x, u_{n}\right)-f(x, u)\right]\left(u_{n}-u\right) d x \longrightarrow 0 \text { as } n \longrightarrow \infty
$$

Observe that

$$
\left\|u_{n}-u\right\|^{2}=\left(\Phi^{\prime}\left(u_{n}\right)-\Phi^{\prime}\left(u_{n}\right)\right)\left(u_{n}-u\right)+\int_{\mathbb{R}}\left[f\left(x, u_{n}\right)-f(x, u)\right]\left(u_{n}-u\right) d x
$$

It is clear that

$$
\left(\Phi^{\prime}\left(u_{n}\right)-\Phi^{\prime}\left(u_{n}\right)\right)\left(u_{n}-u\right) \longrightarrow 0 \text { as } n \longrightarrow \infty .
$$

Combining (3.24) - (3.26), we get $\left\|u_{n}-u\right\| \longrightarrow 0$ as $n \longrightarrow \infty$. The proof of Lemma 3.5 is completed.

Consequently, Lemma 2.3 together with Rermark 2.1 imply that $\Phi$ possesses an unbounded sequence of critical points. Therefore $(\mathcal{F} \mathcal{O D} \mathcal{E})$ possesses infinitely many homoclinic solutions. The proof of Theorem 1.1 is completed.

\section{Proof of Theorem 1.2.}

Lemma 3.6. Under assumptions $\left(\mathcal{A}_{0}\right),\left(F_{1}\right)-\left(F_{3}\right)$ and $\left(F_{5}\right), \Phi$ satisfies the $(C)_{c}$-condition for all $c \in \mathbb{R}$.

Proof. Let $c \in \mathbb{R}$ and $\left(u_{n}\right) \subset E$ satisfying (3.19). First, we prove that $\left(u_{n}\right)$ is bounded in $E$. Arguing by contradiction, suppose that $\left\|u_{n}\right\| \longrightarrow \infty$ as $n \longrightarrow \infty$. Let $v_{n}=\frac{u_{n}}{\left\|u_{n}\right\|}$. Then $\left\|v_{n}\right\|=1$ and $\left\|v_{n}\right\| \leq \eta_{p}\left\|v_{n}\right\|_{L^{p}}$ for $2 \leq p \leq \infty$. By $\left(F_{5}\right)$, one has for $n$ large enough

$$
\begin{gathered}
c+1 \geq \Phi\left(u_{n}\right)-\frac{1}{\mu} \Phi^{\prime}\left(u_{n}\right) u_{n} \\
=\frac{\mu-2}{2 \mu}\left\|u_{n}\right\|^{2}+\int_{\mathbb{R}}\left[\frac{1}{\mu} f\left(x, u_{n}\right) u_{n}-F\left(x, u_{n}\right)\right] d x \\
\geq \frac{\mu-2}{2 \mu}\left\|u_{n}\right\|^{2}-\frac{\rho}{\mu}\left\|u_{n}\right\|_{L^{2}}^{2},
\end{gathered}
$$

which implies

$$
\frac{\mu-2}{2 \rho} \leq \lim \sup _{n \rightarrow \infty}\left\|v_{n}\right\|_{L^{2}}^{2} .
$$

Taking a subsequence if necessary, we may assume that $v_{n} \longrightarrow v$ in $L^{2}(\mathbb{R})$ and $v_{n} \longrightarrow v$ a.e. on $\mathbb{R}$. Hence, it follows from (3.27) that $v \neq 0$. By a similar fashion as for (3.14), we can get a contradiction. Therefore $\left(u_{n}\right)$ is bounded in $E$. The rest of the proof is the same as that in Lemma 3.5 and the proof of Lemma 3.6 is completed

We conclude as in the proof of Theorem 1.1 that $\Phi$ possesses an unbounded sequence of critical points and the proof of Theorem 1.2 is completed.

\section{Conclusion}

Assume that the function $a$ is coercive unnecessary positive and $F(x, u)=\int_{0}^{u} f(x, t) d t$ satisfies some kinds of superquadratic conditions at infinity in the second variable unnecessary satisfying the well-known AmbrosettiRabinowitz superquadratic growth condition., we prove that the fourth-order equation

$$
u^{(4)}(x)+\omega u^{\prime \prime}(x)+a(x) u(x)=f(x, u(x)), \forall x \in \mathbb{R}
$$

possesses infinitely many nontrivial homoclinic solutions by applying critical point theory to the associated least action integral over an appropriate functional space. 
Acknowledgments. The author thanks the referee and the editor for their helpful comments and suggestions.

\section{References.}

[1] C.J. Amik, J.F. Toland; Homoclinic orbits in the dynamic phase space analogy of an elastic struct; Eur. J. Appl. Math. 3 (1991) 97-114.

[2] T. Bartolo, V. Benci, D. fortunato; Abstract critical point theorems and applications to some nonlinear problems with strong resonance at infinity; Nonlinear Analysis, Vol. 7 , No. 9 (1983) 981-1012.

[3] B. Buffoni; Periodic and homoclinic orbits for Lorentz-Lagrangian systems via variational methods; Nonlinear Analysis 26 (1996) 443-462.

[4] J. Chaporova, S. Tersian; Periodic and homoclinic solutions of extended Fisher-Kolmogorov equations; J. Math. Anal. Appl. 260 (2001) 490-506.

[5] P. Coullet, Elphick; D. Repeaux; Nature of spacial chaos; Phys. Rev. Lett. 58 (1987) 431-434.

[6] P.C. Hohenberg, J.B. Swift; Hydrodynamic fluctuations at the convective instability; Phys. Rev. A18 (1977) 319-328.

[7] J. Lega, J.V. Moloney, A.C. Newell; Swift-Hohenberg equation for Lasers; Phy. Rev. Lett. 73 (1994) $2978-2981$.

[8] C. Li; Homoclinic orbits for two classes of fouth-order semilinear differential equations with periodic nonlinearity; J. Appl. Math. Comput. (2008) 27, 107-116.

[9] C. Li; Remarks on homoclinic solutions for semilinear fourth-order ordinary-differential equations without periodicity; Appl. Math. J. Chin. Univ. 24 (2009) 49-55.

[10] F. Li, J. Sun, G. Lu, C. Lv; Infinitely many homoclinic solutions for a nonperiodic fourth-order differential equation without (AR)-condition; Appl. Math. Comput. 241 (2014) 36-41.

[11] F. Li, J. Sun, T-F. Wu; Concentration of homoclinic solutions for some fourth-order equations with sublinear indefinite nonlinearities; Appl. Math. Lett. 38 (2014) 1-6.

[12] F. Li, J. Sun, T-F. Wu; Existence of homoclinic solutions for a fourth-order equation with a parameter; Appl. Math. Comput. 251 (2015)499-506.

[13] S. Lu, T. Zhong; two homoclinic solutions for a nonperiodic fourth-order differential equation without coercive condition; Math. Meth. Appl. Sci. 2016.

[14] T.F. Ma; Positive solutions for a Beam equation on a nonlinear elastic foundation; Math. Comput. Model. 19 (2004) 1195-1201.

[15] P.H. Rabinowitz; Minimax methods in critical point theory with applications to differential equations; CBMS Reg. Conf. Ser. in Math., Vol. 65, American Mathematical Society, Providence, RI, 1986.

[16] Y. Ruan; Periodic and homoclinic solutions of a class of fourth order equations; Rocky Mountain Journal of Mathematics, Vol. 41, No. 3 (2011) 885-907.

[17] J. Sun, T-f. Wu; Two homoclinic solutions for a nonperiodic fourth-order differential equation with a perturbation; J. Math. Anal. Appl. 413 (2014) 622-632.

[18] L. Yang; Infinitely many homoclinic solutions for nonperiodic fourth order differential equations with general potentials; Abstract and Applied Analysis, Vol. 2014, Article ID 435125, 1-7.

[19] L. yang; Multiplicity of homoclinic solutions for a class of nonperiodic fourth-order differential equations with general perturbation; Abstract and Appl. Anal. 2014, Article ID 126435, 1-5.

[20] R. Yuan, Z. Zhang; Homoclinic solutions for a nonperiodic fourth-order differential equation without coercive conditions; Appl. Math. Comput. 250 (2015) 280-286. 